\title{
Lidil
}

Revue de linguistique et de didactique des langues

49 | 2014

L'analyse des données didactiques

\section{Appropriations, médiations et négociations durant un exposé en classe de littérature}

Acquisitions, Mediations and Negociations during a Talk in a Class of French Literature

Appropriazioni, mediazioni e negoziazioni durante una presentazione orale in classe di letterature francese

\section{Chiara Bemporad}

\section{OpenEdition}

\section{Journals}

Édition électronique

URL : http://journals.openedition.org/lidil/3429

DOI : $10.4000 /$ lidil.3429

ISSN : 1960-6052

Éditeur

UGA Éditions/Université Grenoble Alpes

Édition imprimée

Date de publication : 25 mai 2014

Pagination : $33-50$

ISBN : $978-2-84310-272-1$

ISSN : 1146-6480

\section{Référence électronique}

Chiara Bemporad, «Appropriations, médiations et négociations durant un exposé en classe de littérature », Lidil [En ligne], 49 | 2014, mis en ligne le 25 novembre 2015, consulté le 20 avril 2019 URL : http://journals.openedition.org/lidil/3429 ; DOI : 10.4000/lidil.3429 


\title{
Appropriations, médiations et négociations durant un exposé en classe de littérature
}

\author{
Chiara Bemporad*
}

\begin{abstract}
RÉSUMÉ
Le présent article se propose d'analyser la réalisation d'une tâche universitaire en classe par deux étudiantes anglophones apprenant le français comme langue étrangère : un exposé oral effectué dans un cours de littérature française. L'étude observe trois séquences considérées comme des séquences potentielles d'apprentissage qui se sont déroulées lors de l'exposé et complétées par des entretiens oraux effectués à postériori avec les mêmes étudiantes. L'analyse vise à observer la manière dont elles négocient le sens qu'elles donnent aux objets enseignés (la langue, les contenus littéraires et culturels) ainsi que la façon dont elles reconfigurent leurs conduites au sein de la communauté de la classe. De telles négociations situées permettent de relever et étudier des moments spécifiques où des opérations cognitives sont effectuées en vue de l'appropriation de la langue française ainsi que des savoirs spécifiques au cours.
\end{abstract}

\section{ABSTRACT}

This article aims to analyze an academical task in a class by two English students, who learn French as a foreign language: an oral talk made in a course of French literature. The study examines three sequences considered as potential learning sequences that occurred during the presentation. Data are supplemented by oral interviews conducted with the same students after the task. The aim is observing how they negotiate the meaning they give to taught objects (language, literary and cultural contents) and their behaviors within the community of the class. Such situated negotiations allow to identify and examine some specific moments where cognitive operations are performed in order to learn French language as well as specific literary knowledges.

* Université de Lausanne. 
Le présent article se propose d'analyser la réalisation d'une tâche universitaire en classe par deux étudiantes apprenantes du français comme langue étrangère : un exposé oral effectué dans un cours de littérature française. Pendant cette activité, elles négocient le sens qu'elles donnent aux objets enseignés (la langue, les contenus littéraires et culturels), ainsi que leurs conduites à l'intérieur de la communauté de la classe. De telles négociations situées permettent de relever des moments spécifiques où des opérations cognitives sont effectuées en vue de l'appropriation de la langue française, ainsi que des savoirs spécifiques au cours.

\section{Cadre théorique}

Selon une conception socioconstructiviste et sociodiscursive de l'apprentissage, le développement des compétences (langagières, culturelles, etc.) de la personne est situé socialement, historiquement, culturellement et institutionnellement et se réalise dans l'action à travers des opérations de médiation formative (Mondada \& Pekarek Doehler, 2000). Il s'agit d'un processus par lequel les apprenants intègrent les nouvelles informations «aux préconstruits disponibles dans leur environnement socioculturel» (Bronckart, 2004, p. 99).

Les travaux de Lantolf (2000), qui reprennent et actualisent ceux de Bruner (1983), distinguent trois types de médiation : collaborative, individuelle et par les artéfacts. La médiation collaborative (social mediation) peut se dérouler entre un expert et un novice (ici l'enseignant et l'étudiant), ou entre pairs : elle se met en œuvre pendant l'interaction où «un adulte ou un "spécialiste" vient en aide à quelqu'un qui est moins adulte ou spécialiste que lui» en lui permettant de construire des «savoir-faire supérieurs» sur la base de ses «savoir-faire constitutifs » (Bruner, 1983, p. 261-262). Ce type de médiation concerne surtout le développement interpsychique de l'individu. La médiation individuelle (self mediation) est relative au processus du développement intrapsychique; elle prend la forme d'un monologue intérieur ou verbalisé, écrit ou oral. La médiation par les artéfacts (artifact mediation) tient compte du fait que notre développement cognitif s'accomplit aussi à travers la construction, la sémiotisation et l'utilisation d'objets culturels et symboliques comme les textes.

Dans ce travail, il sera question de séquences interactionnelles qui se sont déroulées en classe et peuvent se définir comme des «séquences potentielles d'apprentissage» (SPAP). À la suite des études sur l'acquisition en milieu naturel et guidé (cf. notamment Bange \& Carol, 2005; 
Cambra Giné, 2003; Gajo, Matthey, Moore \& Oesch-Serra, 2004 ; de Pietro, Matthey \& Py, 2004), Moore et Simon (2002) définissent la SPAP comme une «séquence potentiellement acquisitionnelle» (SPA) (de Pietro, Matthey \& Py, 2004) qui s'effectue spécifiquement dans le contexte de la classe. De Pietro, Matthey et Py (2004) ont analysé des séquences dans des interactions exolingues ${ }^{1}$ où le locuteur non natif se focalisait explicitement sur des phénomènes linguistiques spécifiques. Ils ont défini de telles séquences comme des «séquences potentiellement acquisitionnelles» parce qu'elles sont susceptibles d'attirer l'attention de ce locuteur sur des phénomènes langagiers qu'il peut potentiellement intégrer. Il y a ainsi une alternance entre une focalisation de l'attention sur le contenu de la communication et une focalisation sur la forme linguistique. Cette opération est nommée traditionnellement bifocalisation (Bange, 1992, voir aussi Gajo, 2001 et Cambra-Giné, 2003). Les interactions analysées dans l'étude de de Pietro, Matthey et Py se déroulent dans des contextes de socialisation ordinaire et relèvent de l'acquisition langagière, suivant la terminologie de Kraschen (1981) qui oppose l'acquisition, non guidée, à l'apprentissage, guidé par un expert dans un contexte spécifique de formation. Tout en présentant les mêmes phénomènes de bifocalisation, la SPAP de Moore et Simon (2009), au contraire, se déroule dans la communauté de la classe dans un espace institutionnalisé (l'école, l'université, etc.), d'où le terme choisi d'apprentissage. À la suite de Moore et Sabatier (2012), la classe est ici considérée comme une communauté de pratiques (Wenger, 1999) qui «se structure autour de besoins et de buts précis à atteindre et s'inscrit dans le développement de connaissances, de compétences, d'attitudes, voire de valeurs, partagées» (Moore \& Sabatier, 2012, p. 17). Les acteurs ${ }^{2}$ de cette communauté agissent selon «un contrat didactique» (concept défini par Brousseau, 1986, repris par de Pietro, Matthey \& Py, 2004) qui règle leurs interactions et s'attribuent même implicitement des rôles différents, qu'on appellera d'acteur-apprenant et d'acteur-expert.

1. Par exolingue, ces auteurs désignent un échange entre deux locuteurs qui parlent une langue qui n'est pas native pour l'un des deux.

2. Le terme d'acteur renvoie ici à la notion d'acteur social qui, comme le rappellent Kern et Liddicoat (2012), «met en relief la variété des positions du locuteur qui, en tant qu'acteur, peut conjuguer plusieurs modalités d'apprentissage, d'élocution et d'action» (p. 29). 
Moore et Simon (2009) décrivent la SPAP comme «une série d'échanges qui favorise des formatages interactionnels susceptibles de faire travailler les apprenants à l'intérieur de la zone proximale de développement, telle qu'eux-mêmes peuvent la définir et la prendre en charge» (p. 12). La zone proximale de développement (ZPD) ${ }^{3}$ n'étant pas observable en tant que telle, elle peut être inférée à travers les manifestations des opérations cognitives des acteurs-apprenants.

Dans cet article, il sera question d'analyser des séquences potentielles d'apprentissage lors de la réalisation d'une tâche scolaire : un exposé oral sur la lecture et la critique d'une œuvre littéraire. Le terme de tâche est employé ici pour désigner une activité, au sens d'un ensemble d'actions reliées à un même thème (Gajo, 2001, p. 42). Elle est réalisée par un (ou plusieurs) apprenant(s), dans le contexte de la classe, suivant une impulsion donnée par l'enseignant, par le biais de consignes écrites ou orales et d'autres documents fournis à cet effet. Elle renvoie à ce que Puren (2009) considère comme un «agir d'apprentissage », c'est-à-dire une activité effectuée avec l'objectif explicite d'apprendre, en opposition à un «agir d'usage» qui vise à la socialisation ordinaire. La tâche en question poursuit un objectif d'appropriation langagière (améliorer la langue française), culturelle (appréhender certains éléments culturels propres à la culture francophone) et disciplinaire (intégrer des savoirs littéraires spécifiques).

L'analyse des séquences faisant partie de la réalisation d'une tâche permettra d'observer la manière dont les acteurs des interactions négocient le sens qu'ils donnent aux objets traités, ainsi que la manière dont ils reconfigurent leurs conduites au sein de la communauté de la classe, au moment même du déroulement de l'échange conversationnel. Dans ces séquences, il s'agira également d'observer des opérations cognitives qui sont vraisemblablement situées à l'intérieur de la ZPD et qui visent différents types d'appropriation (langagière et disciplinaire). Ces séquences seront reparties selon les différents types de médiation décrits par Lantolf (2000) : collaborative, individuelle et par les artéfacts.

3. Il s'agit de la traduction française de zone of proximal development de l'ouvrage de Bruner (1983). À la suite de Vygotsky (1961) et Bruner (1983), elle est définie comme la distance entre le niveau de développement déterminé à travers la réalisation d'actions de la part d'un apprenant de façon autonome (niveau intrapsychique) et celui déterminé par les actions qu'il réalise lorsqu'il est assisté par un tiers, expert ou pair (niveau interpsychique). 


\section{Méthodologie}

L'analyse concernera deux types de données ${ }^{4}$ : des extraits de l'enregistrement sonore transcrit ${ }^{5}$ d'un exposé oral (qu'on nommera EXP) effectué par deux étudiantes anglophones, Helen (H) et Sophie $(\mathrm{S})^{6}$ et des extraits de deux entretiens oraux (EO) que nous avons menés avec les deux étudiantes, et qui se sont déroulés après l'exposé. La combinaison des pratiques effectives et des pratiques déclarées et reconstruites à postériori par les étudiantes donne accès à leurs représentations ${ }^{7}$ et aux réflexions sur leurs pratiques.

Les séquences conversationnelles analysées se déroulent dans un cours de littérature française adressé à un public d'étudiants qui ne sont pas francophones natifs (niveau B2). Il ne s'agit donc pas d'un cours de langue, c'est-à-dire spécifiquement orienté vers le développement d'un savoir langagier procédural ou métalinguistique, mais d'un cours disciplinaire, c'est-à-dire à contenus culturels et littéraires. Toutefois, le contexte exolingue présuppose toujours une focalisation sur la forme linguistique et stylistique, notamment propre aux genres académiques : le texte littéraire est un produit langagier et culturel complexe dont le traitement et l'appropriation impliquent des compétences langagières et

4. Les données ont été recueillies dans le cadre d'une thèse de doctorat achevée (Bemporad, 2012).

5. La transcription suit les hésitations (ehm), les coupures (mot-), les répétitions et les erreurs des étudiantes. Elle n'est toutefois pas une transcription phonétique (les étudiantes ont un accent anglophone), les pauses sont marquées par un point (.), les emphases par les lettres majuscules.

6. Prénoms fictifs. Ces deux étudiantes anglophones canadiennes sont venues passer une année à Lausanne en 2008-2009, dans le cadre d'un échange universitaire pour suivre des cours de langue, de culture et de littérature française. Elles suivaient un cursus de français dans leur université. Dans le cadre de notre recherche doctorale, nous les avons suivies pendant une année et interviewées à plusieurs reprises par écrit et par oral.

7. Objet de plusieurs études et terme polysémique (voir Py, 2000, 2004 et Moore, 2001, entre autres), la notion de représentation telle qu'elle est utilisée ici se réfère notamment au fait que les étudiantes interviewées reconstruisent dans leur discours une image des objets qu'elles apprennent et des pratiques qu'elles effectuent. Cette image renvoie à des représentations sociales, par rapport auxquelles elles se positionnent, et conditionne leur manière d'apprendre, leurs conduites en classe et leur motivation. Les représentations traitées ici concernent les textes lus, la langue à apprendre ainsi que la tâche que les étudiants interprètent de façon différente. 
métalangagières. C'est pourquoi une tâche de ce genre est particulièrement favorable à l'observation d'épisodes de bifocalisation.

C'est sur le roman de Zola intitulé L'œuvre que porte l'exposé. Ce genre académique oral présuppose un inversement des rôles implicites de la communauté de la classe : les deux étudiantes assument la posture d'expertes du sujet qu'elles doivent présenter aux autres membres de la communauté. Leur expertise est d'ailleurs visible, car elles se positionnent en face de la classe, à la place traditionnellement occupée par l'enseignant qui, lui, reste assis du côté du public - quoique latéralement pour pouvoir plus facilement s'adresser à toute la classe. Helen et Sophie sont donc évaluées de façon formative par leurs pairs qui posent de questions et interviennent pendant l'exposé, et de façon sommative par l'enseignant ${ }^{8}$, qui a de plus le droit, implicite, d'intervenir, de rectifier et de compléter leur exposé, droit qu'il exercera à un moment donné, comme on le verra.

La tâche de l'exposé oral est le résultat de deux types d'activités accomplies par les étudiantes : celles effectuées lors de la préparation et celles inhérentes à la présentation. Si les deuxièmes sont observables et font objet d'analyse, les premières peuvent se reconstruire à partir du résultat, ainsi que des discours des étudiantes, qui ont relaté leurs conduites avant et pendant l'exposé, lors des entretiens oraux. Les activités de préparation consistent essentiellement à la lecture du roman de Zola ainsi qu'à la consultation et la synthèse de lectures secondaires : un texte que l'enseignant a conseillé ainsi que d'autres documents sur la vie de Zola et son rapport avec les peintres impressionnistes que les étudiants ont consultés (principalement sur Internet). L'exposé attendu est une activité de production orale à dominance monologale, incluant des échanges conversationnels mixtes en interaction orale.

À propos du contrat didactique, on peut relever que les acteurs poursuivent trois types d'objectifs : le premier a trait à la réalisation de la tâche explicite, l'exposé, qui consiste en la verbalisation des savoirs préconstruits pour les transmettre à la classe : il s'agit d'une analyse d'un texte littéraire orientée et évaluée par l'enseignant à un moment donné; le deuxième, l'objectif littéraire, concerne la lecture, la compréhension et l'analyse critique du roman de Zola, qui est également objet d'évaluation par l'enseignant au cours de l'exposé; le troisième, l'objectif linguistique, est relatif à l'appropriation du français, qui soustend tout le parcours académique des étudiantes.

8. L'exposé était en effet l'un des travaux requis pour la validation du cours. 
Par l'analyse des données recueillies - des extraits de l'exposé en classe (EXP) et des entretiens oraux (EO) - , il sera possible de saisir la manière dont les étudiants se représentent ces trois objectifs et d'observer les séquences révélant des processus d'appropriation relatifs à ces derniers. Nous observerons des activités caractérisées par une focalisation multiple. En effet, les acteurs ne se borneront pas à faire attention au contenu et à la forme linguistique (la bifocalisation de Bange), mais ils se focaliseront également sur la manière de réaliser la tâche explicite, selon les contraintes propres au genre, sans perdre de vue pour autant les autres objectifs d'appropriation littéraire et/ou linguistique). Il s'agira donc de cerner des moments où les acteursapprenants manifestent des activités mentales de médiation, de réflexion et de négociation.

\section{L'interprétation de la tâche}

Le cours en question a pour thématique «Les romans et les peintres au XIX ${ }^{\mathrm{e}}$ siècle » et vise à développer un savoir disciplinaire sur la littérature et la culture françaises de cette époque. Les étudiantes ont reçu la consigne de décrire les trajectoires des artistes du roman suivant un modèle illustré dans un ouvrage secondaire ${ }^{9}$.

La consigne est contenue dans un chapeau introducteur qui pose la problématique de l'analyse que les étudiants doivent effectuer et consiste pour l'essentiel en la description de l'activité demandée. Ce type de texte "constitue un discours intermédiaire entre l'activité demandée et sa réalisation par les partenaires apprenants» (Cicurel, 2002, p. 5-6).

Le chapeau, qui donne la clé d'analyse du roman, a trait à la comparaison entre deux typologies de carrière des peintres du XIX siècle : l'une, officielle et reconnue par «une organisation institutionnelle très fermée, au service d'un petit nombre de privilégiés», et l'autre, informelle, plus ouverte et non protégée. Cette situation est définie comme «un mélange de protectionnisme institutionnel et de libéralisme juridicosocial $»^{10}$.

9. Il s'agit de Nathalie Heinrich, Être artiste. Les transformations du statut des peintres et des sculpteurs, Paris, 1996, p. 31, 35-34 et chap. 18 et 19.

10. Entre guillemets, les mots de la consigne fournie en annexe, qui renvoient à l'ouvrage cité de Heinrich (1996). 
La consigne mobilise un vocabulaire riche, renvoyant à des concepts complexes présupposant la mise en œuvre de connaissances encyclopédiques et socioculturelles (par exemple : système socioculturel, carrière officielle, protectionnisme institutionnel, libéralisme juridicosocial, consécration du peintre, etc.). Un tel vocabulaire spécialisé peut inciter les étudiants à réemployer ces concepts après se les être appropriés, ce qui sera illustré ensuite.

Après cette introduction, l'énoncé indiquant explicitement la tâche à effectuer est le suivant :

Reconstituez les grandes étapes de la carrière de Claude Lantier et de sa lutte pour la reconnaissance et réfléchissez à ce que chacune d'elles révèle des conditions économiques et socioculturelles auxquelles les peintres de l'époque étaient soumis, et comment elles les représentent et les donnent à comprendre.

La tâche citée présuppose une première lecture globale extensive orientée vers le repérage des passages significatifs relatifs à la carrière du protagoniste, le peintre Claude Lantier; les étudiantes sont appelées ensuite à choisir des extraits à présenter en classe, extraits qui devraient être lus de manière intensive et analytique ${ }^{11}$. Les entretiens avec les deux étudiantes montrent qu'elles n'ont pas la même représentation de cette activité de lecture. Helen, dans son entretien oral, affirme que «la chose plus importante [...] c'était de comprendre bien le livre» et, un peu plus loin, qu' «on avait besoin de comprendre vraiment le thème et tout ensemble» $(\mathrm{EO}-\mathrm{H})^{12}$. L'expression comprendre bien le livre semble suggérer qu'elle se représente une lecture intensive et analytique, ce qui n'est pas vraiment conforme à la tâche qui requérait plutôt une lecture extensive rapide. Sophie, quant à elle, semble décrire une activité plus conforme à celle qui était attendue, car elle décrit une lecture extensive : "parce que pour nous c'était plutôt ehm ehm une sujet général très général pour notre séminaire $(\mathrm{EO}-\mathrm{S})^{13}$.

11. Nous reprenons ici la dichotomie entre lecture extensive et intensive décrite entre autres par Canvat (2007). La première, plus rapide, suit la progression du texte et «convoque une expérience éthico-pratique du monde», alors que la deuxième, plus lente et attentive, souvent pratiquée en classe, requiert un savoir technique, historico-culturel et esthétique (Canvat, 2007, p. 22-23).

12. Entretien oral avec Helen enregistré le 29 mai 2009, dorénavant EO-H.

13. Entretien oral avec Sophie enregistré le 4 juin 2009, dorénavant EO-S. 
Ces témoignages nous permettent de constater dans quelle mesure les représentations et donc l'interprétation de la tâche peuvent varier. On verra comment ces représentations conditionnent le déroulement de ce que les étudiants font pour l'accomplir.

\section{Analyse de trois séquences potentielles d'apprentissage}

Dans cette partie, seront analysées trois séquences conversationnelles se déroulant pendant l'exposé en classe. Pendant la tâche (d'une durée de trente minutes environ), Sophie et Helen alternent leurs prises de parole de façon équilibrée, en s'entraidant. L'enseignant (E) intervient aussi de façon ponctuelle. Les autres étudiants posent des questions et répondent.

Le choix de ces trois séquences, comme on l'a dit, renvoie à différents types de médiation. La première séquence, principalement monologique, illustre en grande partie une activité de médiation individuelle : il s'agit de l'introduction d'Helen, qui résume le livre de Nathalie Heinrich et décrit les différentes trajectoires d'artiste possibles au XIX ${ }^{\mathrm{e}}$ siècle. La deuxième relève d'une médiation collaborative entre pairs : elle illustre l'interaction qui suit la lecture à haute voix d'un extrait faite par Helen, qui a charge de le commenter. La troisième illustre une médiation collaborative apprenant-expert : l'interaction entre les étudiantes et l'enseignant qui intervient pour compléter et rectifier le commentaire de l'extrait. Toute l'activité présuppose une médiation avec des artéfacts (le roman de Zola ainsi que d'autres livres et documents), effectuée avant et pendant l'exposé par les acteurs de la tâche.

\section{Un exemple de médiation individuelle : I'introduction d'Helen}

Dans son monologue, Helen, qui se charge de l'introduction générale, résume le livre de Heinrich et caractérise les différentes trajectoires d'artiste à l'aide d'un schéma qu'elle a dessiné au tableau noir au début du cours. Elle résume deux trajectoires possibles : l'une plutôt conventionnelle et réussie, celle du personnage du peintre Fagerolles, l'autre non conventionnelle et vouée à l'échec, celle de Claude Lantier. Elle introduit son monologue de la façon suivante :

EXP1-H : ok ehm il y a plusieurs trajectoires d'artiste qui sont possibles donc j'ai un schéma ici (elle indique le schéma au tableau noir) qui décrit les les carrières d'un peintre ehm il y a un type un entrée des privilégiés pour suivre une carrière carrière qui est fondée sur l'organisation institutionnelle donc ça veut dire les écoles [...] puis ehm le 
reste comme Claude ils ont suivi le statut informel qui est plutôt ehm pas vraiment enseigné

Par le déictique ici, accompagné du geste de pointer le doigt sur le tableau, Helen adopte des stratégies visant à améliorer l'efficacité communicative. Ses mots reprennent en partie le vocabulaire technique de la consigne qu'elle paraphrase et simplifie. De telles reprises peuvent illustrer comment l'étudiante s'approprie certains mots et concepts. Par exemple, elle reformule et explicite des expressions de la consigne comme «carrières fondées sur l'organisation institutionnelle» ou «statut informel», en explicitant ces termes : «ça veut dire les écoles» (pour organisation institutionnelle) et «pas vraiment enseigné» (pour statut informel).

Helen semble avoir compris les concepts généraux de l'analyse proposée par l'enseignant et s'être approprié le contenu des chapitres du texte de la littérature secondaire et du texte source, qu'elle paraphrase et synthétise. Le recours au schéma dessiné au tableau montre en outre qu'elle a réfléchi à son public.

Son monologue se poursuit avec un apport personnel lorsqu'elle commente les avantages et désavantages de la trajectoire des peintres considérés comme libéraux, en lien avec le concept de consécration de l'artiste :

EXP2-H : puis ehm j'ai fait un petit échelle du succès et puis ehm les professionnels sont ehm ils ont plus de suc- succès parce qu'ils sont plus fameux ils ont plus l'argent et puis ici l'absence de consécration c'était c'est très déprimant on on est pas reçu beaucoup de succès MAIS Alors là les les personnes qui ehm sont libérales dans leurs peintures ehm ils sont plus révolutionnaires parce qu'ils ont pas besoin de suivre les règles mises par ehm l'Acad- l'Académie ehm et donc on peut expérimenter un peu avec le l'art

Helen ne se limite pas à expliquer des modalités de consécration des artistes professionnels et les initiatives aptes à valoriser cette consécration (prix, reconnaissance de l'Académie, etc.), mais elle analyse aussi cette situation et en donne une interprétation personnelle, en soulignant la plus grande liberté des peintres non reconnus. Elle crée un effet de surprise qu'elle introduit par un MAIS Alors là, emphatisé par le ton de sa voix, qui invalide la conclusion implicite négative et renverse la situation initiale en montrant les avantages de la condition des artistes libéraux.

Elle utilise donc des ressources langagières et non langagières (gestuelles, visuelles, vocales) pour améliorer sa communication et compense 
ainsi ses lacunes éventuelles en langue. Malgré ses erreurs linguistiques, sa communication est efficace et son interlangue est cohérente. De plus, elle effectue un travail sur le contenu du chapeau fourni dans le document de la consigne, dont elle reprend des termes, les reformulant et se les appropriant. Son interlangue semble se construire dans un rapport intertextuel avec le chapeau, ce qui dénote une interaction exolingue différée : l'hypotexte écrit (l'introduction de l'enseignant) est réélaboré pour produire l'hypertexte oral. Helen s'approprie des structures et insère de temps en temps des reformulations et des allusions personnelles qui montrent qu'elle a compris et que son interlangue est potentiellement sur le point d'intégrer de telles structures parce qu'elle les reformule : «s'appuyant sur» devient «soutenu par»; les mots et les expressions «vocation», «originalité», «génie individuel» sont paraphrasés par «plus révolutionnaire» ou «on peut expérimenter un peu avec le l'art». Tout en montrant qu'elle a appris de nouveaux mots, qu'elle emploie de façon appropriée, Helen intègre des concepts nouveaux, qu'elle réemploie en vue de mieux interpréter le roman de Zola. Les savoirs sociologiques sur les trajectoires des artistes, qu'elle a intégrés grâce au livre de Heinrich, sont utilisés par Helen et orientent son interprétation du roman.

L'étudiante effectue donc des activités de médiation individuelle en manipulant les textes fournis, et en paraphrasant des éléments. De plus, ses hésitations et reformulations manifestent la perméabilité de son interlangue. Ces éléments nous permettent de constater qu'elle est réceptive vis-à-vis des données langagières et conceptuelles que les textes lui fournissent en vue de réaliser la tâche de l'exposé, et qu'elle est potentiellement en train d'améliorer ses compétences langagières. Sa focalisation est donc multiple : 1) sur la tâche (ou au moins sur sa représentation de la tâche) parce qu'elle essaie de se faire comprendre des autres étudiants par des explications; 2) sur la langue, parce qu'elle reformule les phrases et réfléchit aux termes et aux structures grammaticales; et 3) sur le contenu disciplinaire lorsqu'elle est capable de sélectionner les éléments du texte et d'expliquer le comportement des personnages de l'histoire en fonction d'une interprétation personnelle conditionnée par une lecture secondaire. Il est donc possible d'observer un développement potentiel de ses compétences langagières et littéraires. 


\section{Un exemple de médiation collaborative : le commentaire du texte}

La deuxième séquence concerne le commentaire d'un extrait du roman que les étudiantes ont choisi pour illustrer les situations décrites en introduction. Helen situe d'abord l'extrait dans le cadre général du roman : le tableau de Claude exposé au salon, qui représente son enfant mort, est suspendu en haut du mur, complètement caché au regard du public. Ensuite, elle lit le passage à voix haute et essaye d'impliquer les autres étudiants en leur proposant un exercice de repérage des mots; elle demande : «est-ce qu'on veut ensemble trouver les mots qui ehm donnent l'impression [...] de la l'échec les mots reliés à le l'échec».

C'est à ce moment-là qu'une difficulté de compréhension surgit, car une étudiante demande une explication du mot échec, ce qui illustre un épisode de focalisation multiple :

EXP3 : Étu1 : Qu'est-ce que ça veut dire ehm échec?

H2 : l'échec c'est ehm.

S3 : faillite

$\mathrm{H} 4$ : faille?

Étu5 : ah oui oui

E6 : le fait de ne pas réussir

Étu7 : oui

E8 : c'est le contraire de la réussite l'échec.

S9: des mots négatifs en général peut-être.

Dans cette séquence, il y a alternance de quatre interlocuteurs : Helen, Sophie, l'étudiante qui ne connait pas le mot «échec» et l'enseignant (E). On voit ici qu'on se trouve face à une triple négociation. D'abord, il y a une négociation sur la signification du mot échec qui dénote un épisode de bifocalisation selon Bange (1992), entre message et forme : Helen essaie de répondre, mais Sophie lui vient en aide en proposant le synonyme («faillite») (S3). Ce terme est clair pour l'étudiante (Étu5 et Étu7), mais pas pour Helen qui le confond avec «faille» (H4). La négociation continue avec l'intervention de l'enseignant qui explique la signification du mot en recourant à son antonyme (E6 et E8). Lorsque Sophie reprend la parole, toutefois, elle change de plan en reformulant non pas la signification du mot échec, mais la consigne (S9), pour permettre à la classe d'effectuer l'exercice proposé d'analyse du texte (repérer les mots relatifs à l'échec).

Cette séquence montre une alternance entre une négociation linguistique, plus spécifiquement sémantique, une négociation pragmatique qui vise l'accomplissement de l'activité demandée aux étudiants et une 
négociation littéraire, c'est-à-dire focalisée sur la lecture et l'interprétation des morceaux choisis par les étudiantes. Cette dernière négociation s'oriente vers une analyse stylistique autour de l'évocation des mots relatifs à l'échec.

Par ailleurs, bien que l'enseignant intervienne, il assume le même rôle que les autres étudiants, en se positionnant en tant que pair, et c'est Sophie qui clôt la séquence en recentrant les étudiants sur la tâche et en assumant le rôle d'experte.

\section{Séquence de médiation collaborative avec expert : complément de l'analyse littéraire}

Après l'activité de repérage des mots, Helen et Sophie proposent une interprétation du texte. Elles invitent à considérer les termes se rapportant à l'échec et à la mort utilisés pour décrire le tableau de Claude comme une anticipation du suicide de l'artiste à la fin du livre :

EXP4-S : et ehm je pense que ça c'est vraiment comme comme c'est c'est décrit plus tard c'est la MORT de sa carrière et c'est comme une métaphore son enfant est mort et sa carrière est mort avec c'est un un œuvre qui décrit la mort et c'est aussi l'œuvre qui qui a le résultat de tuer son carrière c'est pas c'est pas tué mais son son carrière est aussi tuée mort

$\mathrm{H}$ : donc juste le résumé de ça c'est que sa carrière est un échec et aussi ehm la famille d'avoir un fils ça il a aussi échoué d'être une père parce que n'est pas je sais pas son enfant est malade et il est mort you know.

Sophie et Helen cherchent ici à transmettre une interprétation du contenu du roman, qui se base sur une analyse formelle, notamment sémantique. Elles explicitent le lien entre le tableau de l'enfant mort et le suicide du peintre, en citant les mots du texte. Sophie parle en effet de «métaphore», terme technique qui lui permet de justifier le lien qu'elle fait entre la mort (mot qu'elle emphatise à l'oral) de l'enfant, la carrière et l'artiste lui-même. De la même façon Helen, en reprenant le mot échec, précédemment discuté avec les autres étudiants, rebondit sur l'interprétation de Sophie en soulignant également la faillite de la paternité de Claude Lantier (à noter l'emploi d'échec et d'échouer).

Par ailleurs, après la synthèse de cette interprétation, l'enseignant demande d'intervenir et apporte des données textuelles qui soulignent ultérieurement que l'échec de Claude est dû au fait que personne ne peut voir son tableau : 
EXP5-E1 : je crois que effectivement juste après ce passage si ça [...] c'est vrai que le tableau lui-même ehm l'IMAge du tableau c'est l'enfant mort donc les les termes d'échec que vous avez souligné quelque part il les a assumés en entier [S : mm] parce qu'il a peint son enfant mort mais il y a un autre échec [...] qui fait écho avec cette mort qui est présentée c'est le fait que personne ne voit le tableau [...] c'est JUSTE après votre passage [...] (il lit le texte à haute voix) c'est là qu'il y a la phrase dont tout à l'heure vous parliez

S2 : oui c'était la mort

$\mathrm{E} 3$ : je trouve que c'est important de citer tout le passage (il lit le texte à voix haute) donc le fait qu'il y a même qu'il n'y a pas de public même pour le DETESter (il sourit) [...] là il n'y a même plus de contact ehm le tableau PERSONNE ne le voit et ça c'est la vraie mort hem donc il y a il y a une trajectoire avec

S4 : parce que oui il n'attendait il n'attendait pas le succès en fait il attendait vraiment que les personnes ehm

E5 : soient scandalisées

S6 : oui soient scandalisées

E7 : mais il ne s'attendait pas à ce qu'il y ait personne qui voie son tableau.

Dans ce passage, l'enseignant donne une interprétation plus subtile et élaborée que celle des étudiantes et souligne que leur argumentation aurait été plus efficace si elles avaient cité la suite du passage, où ce qu'elles avaient dit de façon implicite s'explicitait. Sophie semble comprendre ce que l'enseignant veut dire, lorsqu'elle anticipe la phrase du texte que l'enseignant est en train de lire (S2). Les interventions de Sophie complètent le raisonnement de l'enseignant en engageant une négociation de l'interprétation (S4 et S6). Ce processus de construction d'un savoir est un exemple de médiation apprenant-expert; l'enseignant agit alors au sein de la zone proximale de développement de Sophie.

Sophie commente cet épisode dans l'entretien oral :

EO-S : j'avais pas probablement pas compris [...] que c'était vraiment important [...] parce que je pense que j'ai j'ai lu pour savoir le contexte si j'avais lu quelque page quelque page avant ou après le passage mais je je voulais juste choisir quelque chose court.

En affirmant n'avoir pas compris, Sophie se réfère alternativement au texte (donc à l'objectif littéraire de la lecture du roman) et à la réalisation de l'exposé, ce qui montre une focalisation multiple et une activité de médiation complexe. 
Helen aussi, dans son entretien oral, commente ce moment de l'exposé et en cherche une explication : «oui parce que on a pris une citation et puis il a dit mais vous avez manqué le la partie la plus important qui vient juste après». Helen montre ici qu'elle s'est aperçue de l'importance de ce passage, ce qui permet de voir comment l'intervention de l'enseignant étaye leur raisonnement et leur interprétation, en améliorant leur interprétation du texte.

On comprend mieux l'engagement de Sophie lors de l'interaction avec l'enseignant en classe : elle voulait peut-être rattraper un manque ressenti intuitivement pendant la préparation de l'exposé.

Cet épisode provoque la sensation d'une faiblesse de la part des deux étudiantes qui perçoivent leur tâche comme non complètement réussie. Notons que les reformulations des mots de l'enseignant ne correspondent pas exactement à ce qu'il a dit : l'enseignant avait introduit sa remarque de façon neutre (EXP5-E3 : «je trouve que c'est important de citer tout le passage»); Sophie emphatise la phrase (EO-S : «j'avais pas compris que c'était vraiment important»); Helen exagère ultérieurement (EO-H : «il a dit mais vous avez manqué le la partie la plus important»). Les entretiens nous montrent que les étudiantes reconstruisent après coup leur réalité. De plus, ils nous permettent de voir qu'il y a eu une intériorisation du raisonnement effectué par l'enseignant en classe. En effet, Sophie et Helen montrent qu'elles comprennent la remarque de l'enseignant et sont d'accord avec lui, c'est-à-dire que ces remarques sont à leur portée et situées dans leur ZPD : cette médiation collaborative agit sur l'appropriation disciplinaire.

\section{Conclusion}

L'analyse de ces trois séquences d'apprentissage permet de tirer des conclusions à la fois méthodologiques et didactiques.

Au plan méthodologique, elle souligne l'importance des analyses des pratiques de classe qui permettent de mettre en œuvre un dispositif de recherche offrant la possibilité de revenir sur les actions effectuées pour observer le processus d'appropriation des acteurs-apprenants. Par le biais d'une analyse approfondie à postériori, il est possible de séparer et de catégoriser des activités effectuées simultanément. Par ailleurs, compléter les données des pratiques de classe avec des entretiens oraux donne accès aux représentations, aux reconfigurations et aux réflexions des acteurs. 
Les analyses des différentes médiations et focalisations ont permis d'identifier des moments où l'apprenant est en train d'agir sur sa ZPD et donc potentiellement en train d'apprendre. C'est effectivement dans ces moments de négociation que l'enseignant peut agir.

Cette étude permet en outre de faire des observations sur l'enseignement de la littérature en contexte exolingue, aspect encore peu étudié. Un tel contexte, en effet, est particulièrement propice à susciter des activités de focalisation multiple et alternée portant sur des objectifs à la fois linguistiques et littéraires. Lors de l'exposé, Sophie, Helen et les autres étudiants de la classe se sont interrogés sur les structures et sur les termes linguistiques du texte pour effectuer une interprétation littéraire du roman, et en même temps pour apprendre de nouveaux mots. Nous pouvons définir la tâche comme littéraire, dans la mesure où les étudiantes ont sélectionné et commenté des passages pour avancer des interprétations du roman. L'une de ces interprétations était sociologique (les trajectoires des artistes au XIX ${ }^{\mathrm{e}}$ siècle) et a été suggérée par l'enseignant qui leur a fourni une clé de lecture à travers le livre de Heinrich; une autre était relative au lien entre le contenu général du roman et des passages choisis évoquant la mort et l'échec d'un personnage; cette interprétation a été avancée par les étudiantes de façon personnelle et étayée par l'enseignant au moment de l'exposé en classe. Cette activité d'analyse critique a été effectuée par les étudiantes en même temps qu'elles amélioraient leur interlangue : elles ont appris en faisant; elle s'est faite en outre en fonction de cette amélioration, car elles n'ont pas cessé de se focaliser à la fois sur le message et la forme, en apprenant et employant des mots qu'elles auraient pu réutiliser plus tard. Enfin, les propos avancés par les étudiantes lors de leur entretien montrent qu'elles ont compris leurs lacunes et erreurs, grâce aux remarques de l'enseignant. Il est donc possible d'affirmer que cette tâche était située dans leur zone proximale de développement.

Pour conclure, les observations sur les processus de médiation des apprenants illustrent combien l'appropriation est un processus dynamique et coopératif dans le sens donné par Kress (2001) (voir aussi Filliettaz \& Bronckart, 2005) : dynamique, parce que les activités de médiation sont multiples et évolutives, coopératif, car les nouveaux acquis se construisent dans l'échange, la communication et l'interaction. 


\section{RÉFÉRENCES BIBLIOGRAPHIQUES}

BANGe, Pierre. (1992). À propos de la communication et de l'apprentissage de L2 (notamment dans ses formes institutionnelles). AILE, 1. Disponible en ligne sur $<$ http://aile.revues.org/4875> (consulté le 4 mars 2014).

BANGE, Pierre \& CAROL, Rita. (2005). L'apprentissage d'une langue étrangère : cognition et interaction. Paris : L'Harmattan.

BEMPORAD, Chiara. (2012). Lectures littéraires d'étudiant.e.s plurilingues et appropriations langagières (Thèse de doctorat). Université de Lausanne.

Brousseau, Guy. (1986). Fondements et méthodes de la didactique des mathématiques, Recherche en didactique des mathématiques. Grenoble : La Pensée sauvage, 1986.

BRONCKART, Jean-Paul. (2004). Les genres de textes et leur contribution au développement psychologique. Langages, 153(1), 98-108.

BRUNER, Jérôme. (1983). Le développement de l'enfant : savoir faire, savoir dire. Paris : Presses universitaires de France.

CAmbra Giné, Margarita. (2003). Une approche ethnographique de la classe de langue. Paris : Crédif-Didier.

Canvat, Karl. (2007). Lire du côté de chez soi. Réhabiliter la lecture «ordinaire». Dans C. Bemporad \& T. Jeanneret (dir.), Lectures littéraires et appropriation des langues étrangères (vol. 4, p. 19-52). Lausanne : Études des Lettres.

Cicurel, Francine. (2002). La classe de langue un lieu ordinaire, une interaction complexe. AILE, 16. Disponible en ligne sur $<\mathrm{http}$ ://aile.revues. org/74> (consulté le 4 mars 2014).

De Pietro, Jean-François, Matthey, Marinette \& Py, Bernard. (2004). Acquisition et contrat didactique : les séquences potentiellement acquisitionnelles dans la conversation exolingue. Dans L. Gajo, M. Matthey, D. Moore \& C. Serra (dir.), Un parcours au contact des langues. Textes de Bernard Py commentés (p. 79-93). Paris : Didier.

Filliettaz, Laurent \& Bronckart, Jean-Paul (2005). L'analyse des actions et des discours en situation de travail : concepts, méthodes et applications. Louvain-la-Neuve : Peeters.

GAJO, Laurent. (2001). Immersion, bilinguisme et interaction en classe. Paris : Didier.

Gajo, Laurent, Matthey, Marinette, Moore, Danièle \& Oesch-Serra, Cecilia (dir.). (2004). Un parcours au contact des langues. Textes de Bernard Py commentés. Paris : Didier.

Krashen, Stephen D. (1981). Second Language Acquisition and Second Language Learning. Oxford, New York : Pergamon Press. 
Kress, Gunther R. (2001). Multimodal Teaching and Learning: The Rhetorics of the Science Classroom. Londres, New York : Continuum.

Lantolf, James P. (2000). Second Language Learning as a Mediated Process. Language Teaching, 33, 79-96.

Mondada, Lorenza \& Pekarek Doehler, Simona. (2000). Interaction sociale et cognition située : quels modèles pour la recherche sur l'acquisition des langues? AILE, 12. Disponible en ligne sur <http://aile.revues. org/74> (consulté le 4 mars 2014).

Moore, Danièle \& SABATIER, Cécile. (2012). Une semaine en classe en immersion française au Canada : approche ethnographique pour la formation. Grenoble : Presses universitaires de Grenoble.

Moore, Danièle \& Simon, Diana-Lee. (2002). Déritualisation et identité d'apprenant. AILE, 16. Disponible en ligne sur <http://aile.revues.org/ 74> (consulté le 4 mars 2014).

Puren, Christian. (2009). Variations sur le thème de l'agir social en didactique des langues-cultures étrangères. Recherches et applications, 45, $1-38$.

Py, Bernard. (2004). Pour une perspective bilingue sur l'enseignement et l'apprentissage des langues. Dans L. Gajo, M. Matthey, D. Moore \& C. Serra (dir.), Un parcours au contact des langues. Textes de Bernard Py commentés (p. 139-146). Paris : Didier.

Wenger, Étienne (dir.). (1999). Communities of Practice: Learning, Meaning, and Identity. Cambridge : Cambridge University Press.

Vygotsky, Lev Semenovič. (1962). Thought and Language. Cambridge, Mass. : The M.I.T. Press. 